\title{
Dossier Évolution et créationnisme Génétique moléculaire et évolution
}

\author{
Jacqueline Laurent \\ Maître de conférences en génétique moléculaire et cellulaire, Université Paris Sud 11, Faculté des sciences d'Orsay, \\ Institut de génétique et microbiologie, bât. 409, 91405 Orsay cedex, France
}

\section{Le choix de la génétique}

Faire de la recherche expérimentale est un projet auquel j'ai aspiré encore très jeune, le choix de la génétique est venu après. Il correspondait à un compromis entre les mathématiques et la biologie, entre la rigueur des raisonnements logiques, d'une part, et la curiosité suscitée par la richesse de la nature, sa diversité, son invention créatrice, d'autre part. Au moment de ce choix, je ne percevais pas encore toute la complexité du vivant et le défi que cela représentait de prétendre élucider (une partie de) son fonctionnement à travers un sujet de recherche. Aujourd'hui, lorsque je fais le bilan au terme de ma vie professionnelle d'enseignant-chercheur en génétique, il m’apparaît que la théorie de l'évolution, qui explique parfaitement cette exubérance, a été, explicitement ou implicitement, une référence constante pour mes travaux de recherche comme pour mon enseignement. J'espère le montrer dans ce qui suit.

\section{La phylogénie moléculaire et l'évolution à la lumière des gènes et des mutations}

Les concepts-clés de l'évolution sont explicitement à la base des travaux de phylogénie moléculaire que j'ai effectués ces onze dernières années (Philippe et Laurent, 1998 ; Philippe et al., 2000 ; Filée et al., 2002 ; Filée et al., 2003). L'hypothèse fondamentale de la phylogénie moléculaire est que le nombre de mutations accumulées au cours des générations est d'autant plus important que

Auteur correspondant : faline_jl@aliceadsl.fr

Voir aussi les autres contributions au dossier «Évolution et créationnisme " dans le Vol. 15, $\mathrm{n}^{\circ} 2$ et suivants (rubriques Éditorial et Forum). les organismes considérés sont évolutivement éloignés (on parle de distance). Cette hypothèse a d'abord été validée sur des groupes d'organismes dont les relations phylogénétiques avaient été établies par ailleurs sur la base de données d'anatomie comparée. Ensuite, grâce à une intensification des programmes de séquençage des génomes, des études poussées, basées uniquement sur des données de séquences, ont permis d'établir les relations phylogénétiques entre des espèces difficilement classifiables sur d'autres critères.

Sans la théorie de l'évolution, ces travaux n'auraient eu a priori aucune justification, et, dans ce cas, de deux choses l'une : soit ils n'auraient pas été initiés, soit ils auraient été à l'origine d'une réflexion qui aurait très probablement conduit à énoncer... une théorie de l'évolution!

\section{L'évolution : son statut, ses mécanismes}

\section{L'évolution, un fait scientifique}

Sur le plan historique, la théorie de l'évolution a joué un rôle essentiel. Quand il est apparu, au XIX ${ }^{\mathrm{e}}$ siècle, le concept d'évolution recouvrait un ensemble de théories : les théories transformistes, dont les principales sont le lamarckisme (transmission des caractères adaptatifs acquis pour répondre à des instructions de l'environnement), et le darwinisme (variation, sélection des individus adaptés aux conditions de l'environnement). À l'époque, il s'agissait d'hypothèses capables d'expliquer des observations, mais, en l'état des connaissances sur les mécanismes de l'hérédité, ces hypothèses ne pouvaient reposer sur aucun support matériel tangible.

Partant de l'observation et de la comparaison à différents niveaux d'individus appartenant à diverses espèces, la démarche scientifique conduit à décrire des mécanismes 
qui expliquent que les êtres vivants sont le résultat de l'évolution. L'évolution serait donc un objet que construit une science, autrement dit un fait scientifique.

\section{Les mécanismes de l'évolution}

\section{L'évolution selon Darwin}

Telle qu'exprimée par Darwin (1859), dans De l'origine des espèces, la théorie de l'évolution, par transformation graduelle, repose sur la sélection naturelle, au sein d'une population diversifiée, des individus les mieux adaptés à leur environnement à la fois pour la survie et pour la reproduction, c'est-à-dire, finalement, pour leur capacité à donner une descendance. Les descendants des individus ainsi « sélectionnés » héritent de certaines caractéristiques de leurs parents et les transmettent à leur propre descendance. En augmentant la diversité, ces modifications successives améliorent sans cesse l'efficacité d'adaptation à d'éventuels changements d'environnement. Les moteurs de l'évolution sont donc : «descendance avec modification » (mutation au sens large) et sélection. Au niveau macroscopique, on a pu montrer que la sexualité et l'isolement géographique ou les migrations ont joué un rôle non négligeable dans l'augmentation de la diversité et l'apparition de nouvelles espèces.

\section{Une base physico-chimique pour l'évolution}

Outre les données anatomiques sur lesquelles étaient basées les premières hypothèses de la théorie de l'évolution, nous disposons maintenant de données cellulaires et moléculaires. Les avancées scientifiques concernant la nature du matériel génétique et le fonctionnement des gènes supports de l'information héréditaire ont conforté la théorie de l'évolution en lui donnant une base physico-chimique. Le matériel génétique a deux propriétés remarquables essentielles, tant pour assurer la reproduction que pour produire de la biodiversité : il peut se reproduire avec exactitude et/ou subir des changements soudains, spontanés ou non, de sa séquence (par mutation) ou de sa structure (par mutation ou par des modifications épigénétiques ${ }^{1}$ de l'ADN ou de la chromatine).

\section{Apports de la phylogénie moléculaire}

Ce sont les outils de la phylogénie moléculaire qui ont permis de démontrer que tous les êtres qui vivent actuellement sont issus d'un même ancêtre commun (résultant

\footnotetext{
${ }^{1}$ Les modifications épigénétiques sont des modifications chimiques (méthylations, phosphorylations...) de certains nucléotides de l'ADN ou de certains acides aminés des protéines associées aux chromosomes. Ces modifications régulent l'expression des régions génomiques.
}

lui-même d'un processus évolutif préalable). De fait, tous les êtres vivants actuels sont le résultat de processus évolutifs tout aussi anciens, ils sont donc tous aussi évolués les uns que les autres. Par suite de la diversification évolutive, les êtres vivants présentent des caractéristiques qui permettent de les regrouper en sous-ensembles de plus en plus grands, jusqu'à définir trois grands domaines : les archées, les bactéries et les eucaryotes.

On démontre que des échanges de matériel génétique se font par transfert horizontal, y compris entre des organismes appartenant aux différents domaines, soit par le biais du parasitisme ou de la symbiose, soit par l'intermédiaire de virus ou de transposons.

Les connaissances actuelles sur les génomes permettent désormais d'expliquer non seulement l'apparition de fonctions nouvelles par accumulation de mutations, mais aussi celle des organismes très divers qui ont colonisé tous les écosystèmes de notre planète, par des mécanismes de transfert horizontal, de remaniement chromosomique et/ou de changement de l'état d'expression des gènes.

\section{Un sujet de thèse qui se réfère explicitement à l'évolution}

De fait, sans l'adhésion au concept d'évolution, le sujet même de ma thèse, commencée en 1969, ne m'aurait jamais été proposé. En effet, l'objectif qui m’était assigné consistait à analyser les modifications du VSV (virus de la stomatite vésiculeuse) après son inoculation à un nouvel hôte.

Le VSV appartient à la famille des rhabdovirus, il est à l'origine d'une maladie infectieuse des bovins auxquels il est transmis, via des piqûres d'insecte, à partir de populations virales « réservoir » chez des vertébrés (chauve-souris, par exemple). Mon travail consistait à comparer différentes caractéristiques de populations de VSV multipliées soit dans des cellules de vertébré, soit dans des drosophiles (la drosophile est l'hôte du virus sigma, sujet « historique » d'étude au laboratoire) après inoculation de ces insectes. Par différentes approches, j'ai pu montrer que tout se passait comme si, au fur et à mesure des passages, le virus s'adaptait à son nouvel hôte tout en se désadaptant de son hôte d'origine (Laurent, 1983 ; Laurent et Lafay, 1983 ; Laurent, 1984) : une illustration parfaite du processus de sélection, au sein d'une population virale, des particules les plus adaptées à l'hôte dans lequel elles se trouvent.

\section{À la recherche d'un gène à partir d'un de ses cousins supposés}

Par ailleurs, je me suis intéressée aux facteurs génétiques déterminants du pouvoir pathogène d'Erwinia amylovora, bactérie phytopathogène, responsable du feu bactérien. 
Après avoir produit et sélectionné des mutants avirulents par des techniques de génétique moléculaire, nous avons identifié les régions génomiques correspondantes. Pour un type de mutants avirulents, nous voulions savoir si les gènes concernés étaient apparentés à ceux, pris comme référence, d'une autre bactérie présentant des caractéristiques proches (Dolph et al., 1988; Coplin et al., 1992; Menggad et Laurent, 1998).

Pour cela, deux approches basées sur nos connaissances de l'évolution des séquences des gènes, sont concevables :

- l'hybridation de séquences, qui suppose que les séquences de deux gènes homologues (issus d'un même gène ancestral) ont conservé suffisamment de similitudes pour pouvoir se réassocier en formant des molécules «hybrides";

- la complémentation fonctionnelle, qui consiste à introduire un fragment $\mathrm{d}^{\prime} \mathrm{ADN}$ contenant des gènes de référence d'une espèce dans des cellules d'un mutant de l'espèce étudiée. Si le gène introduit assure une fonction semblable à celle du gène muté de la souche réceptrice, il peut être capable de corriger le caractère mutant de la souche réceptrice, même si les séquences des gènes sont dissemblables (homologues ayant beaucoup divergé, ou non-homologues).

Dans notre cas, seule l'expérience de complémentation (Bernhard et al., 1993 ; Bugert et Geider, 1995) a donné des résultats positifs. Il a été confirmé par la suite que les deux espèces examinées ne partagent qu'un faible nombre de gènes homologues pour les fonctions étudiées.

Dans cet exemple, les protocoles expérimentaux utilisaient la connaissance des mécanismes de l'évolution en relation avec la structure et le fonctionnement du génome.

\section{Une vision moderne de l'évolution}

\section{Hasard et gènes mutateurs : une assurance pour la vie}

Le modèle hasard-sélection rend compte du caractère aléatoire des événements mutationnels qui touchent n'importe quelle partie d'un génome à n'importe quel moment (Gayon, 1997; Kupiec et Sonigo, 2000). Le caractère aléatoire des phénomènes qui contribuent à la diversité biologique implique deux propriétés importantes :

- le hasard n'est pas assimilable à une absence de cause(s) : l'existence d'agents mutagènes suffit pour illustrer ce point. Même les mutations dites spontanées ont une cause, ne serait-ce qu'une erreur de réplication non réparée (Laurent, 1997);

- par contre, l'idée de hasard suggère une absence de but. On sait depuis les travaux de Luria et Delbrück (1943) que les mutations n'ont aucun caractère adaptatif : elles ne sont pas induites, mais révélées par les conditions dans lesquelles s'exprime un caractère mutant.

Il est admis qu'aucune finalité (au sens d'un projet précis, d'une intention) ne commande le processus évolutif, et qu'une mutation n'est le plus souvent ni favorable ni défavorable. Son effet dépendra des conditions dans lesquelles se trouve l'individu affecté, puisque c'est sur l'organisme que s'exerce la sélection.

La théorie neutraliste de l'évolution moléculaire, énoncée par Kimura en 1967 ( $c f$. Kimura, 1997), est un apport de l'approche moléculaire et statistique. Cette théorie suggère que la plupart des substitutions de nucléotides seraient sélectivement neutres et seraient maintenues dans les populations par un équilibre entre leur taux de production par mutation et leur élimination aléatoire.

Le concept de "gènes mutateurs " est né d'une réflexion sur l'équilibre entre les coûts de la mutabilité (mutations délétères) et ses bénéfices (optimisation de l'adaptabilité). Puisque la diversité est essentielle au maintien de formes de vie et que les mutations sont nécessaires pour générer cette diversité, il est vraisemblable que la capacité à muter ait été sélectionnée positivement au cours de l'évolution. L'apparition de la plupart des mutations résulte d'erreurs (rarissimes) lors du processus de réplication de l'ADN, associées à une déficience des systèmes de détectionréparation de ces erreurs (Laurent, 1997 et 2003). Comme les autres, les gènes responsables du maintien de l'intégrité des génomes sont susceptibles de muter, ce qui entraîne alors une augmentation de la mutabilité de l'ensemble du génome. On observe aussi que la capacité à muter persiste dans la nature, notamment sous forme de systèmes dont les exécutants sont des protéines, les ADN mutases, capables de répliquer, sans matrice, de l'ADN endommagé et donc... d'inventer de la nouveauté.

Parallèlement, des stratégies de contrôle du coût de la mutagenèse adaptative se sont développées (Radman et al., 2000 ; Sniegowski et al., 2000). Leur rôle est de restreindre l'augmentation du taux de mutation, soit à des régions précises du génome (hypermutabilité de gènes particuliers, comme ceux du système immunitaire des animaux), soit à des moments particuliers (déclenchement de l'activité de systèmes mutateurs dans des conditions de stress environnemental). Les systèmes mutateurs font partie des systèmes de régulation fine, comme ceux qui régissent la physiologie d'une cellule ou d'un organisme.

\section{L'évolution, un équilibre subtil entre continuité et innovation}

À la lumière des connaissances actuelles, l'évolution des êtres vivants apparaît comme un compromis entre continuité et innovation. La stabilité du génome et sa transmission fidèle à la descendance assurent le maintien de la vie dans un environnement stable. Dans ces conditions 
« normales », des formes variantes des gènes apparaissent néanmoins ; celles-ci sont ensuite retenues ou éliminées sous la pression de la sélection. Par contre, dans certaines situations extrêmes, ce sont les conditions environnementales (la pression de sélection) qui provoquent une forte augmentation des événements (mutation et recombinaison) générateurs de nouveaux variants, dont certains seront plus aptes que les autres à survivre dans ce nouveau contexte (Laurent, 1997). Tout se passe alors comme si la pression de sélection était elle-même à l'origine de mutations, faisant en sorte que l'efficacité pour la survie d'au moins quelques individus l'emporte sur la fidélité génétique (Laurent, 2003). De fait, comme elle le fait sur toutes les fonctions biologiques, la sélection naturelle agit aussi sur des mécanismes qui génèrent et modulent la diversité biologique en régulant l'équilibre stabilitémutabilité. Les systèmes de régulation fine sélectionnés au cours de l'évolution permettent d'apporter des réponses adaptées aux changements, y compris à des changements imprévisibles tels que ceux qui résultent des impacts du monde vivant sur le milieu qui l'entoure.

\section{L'évolution, un concept opérationnel unificateur}

À la lumière des connaissances actuelles, l'évolution est l'hypothèse scientifique la plus simple pour expliquer la diversité du monde vivant. Elle permet de comprendre comment chaque être vivant est le résultat d'une succession de mises au point progressives (bricolage) de systèmes biologiques complexes, de mieux en mieux adaptés et même adaptables aux changements (processus hormonaux, réponse immunitaire, gènes mutateurs...). Les nouvelles données, loin de le remettre en cause, confortent régulièrement le concept d'évolution, efficace pour expliquer le vivant. En outre, l'évolution, fait scientifique, est un concept opérationnel unificateur pour les sciences de la nature, tout particulièrement pour les sciences biologiques (Smocovitis, 1992) jusqu'à la biologie du vieillissement et de la mort (Klarsfeld et Revah, 2000).

\section{L'évolution dans le monde contemporain}

L'évolution, qui associe unité, diversité et adaptabilité, est l'essence même du monde vivant : dès que la vie est apparue, sous une forme encore incertaine en l'état actuel des connaissances, elle a commencé à évoluer. Pour que la vie continue, il suffit de lui laisser le temps d'évoluer et de s'adapter.

Aussi la responsabilité de l'homme, des scientifiques en particulier, est-elle grande, car c'est le seul être vivant sur Terre qui ait la connaissance de ces processus et de leur lenteur. Lorsque les processus s'accélèrent, ils sont sources d'inventions nouvelles, mais au détriment de formes de vie actuelles. C'est l'homme qui sait tout cela! Et c'est lui qui a les actions les plus dramatiques sur l'environnement et sur toutes les formes de vie (y compris la vie humaine).

Les scientifiques, conscients de l'échelle de temps nécessaire aux mécanismes d'adaptation aux changements, ont l'énorme responsabilité de le faire savoir.

\section{Évolution versus création, la responsabilité des scientifiques}

Science et croyance ont probablement une origine commune : les interrogations des hommes devant les mystères de la nature, de la vie, de l'homme - cet animal si particulier qui est en mesure de saisir et d'analyser ces mystères. Elles relèvent néanmoins de deux registres différents de la nature humaine : la pensée rationnelle, d'une part, et l'adhésion instinctive à des affirmations non vérifiables, d'autre part.

Comme science et croyance, évolution et création ne relèvent pas des mêmes registres. L'évolution est un fait scientifique élaboré par la pensée rationnelle dans le cadre de la démarche expérimentale, alors que la création décrite dans les textes sacrés de tradition orale est présentée comme une vérité révélée. C'est une allégorie, une explication poétique de l'apparition de la Terre et de la vie sur Terre, géocentriste et « anthropotrope », qui n'a rien de scientifique.

Même si science et croyance peuvent cohabiter chez une même personne, elles ne doivent en aucun cas se superposer sous peine de confusion (Delsol, 1997 ; Salomon, 2006).

La démarche scientifique nous indique que l'on peut (doit) conserver une hypothèse interprétative, simple si possible, tant qu'elle est compatible avec l'observation et les résultats de l'expérience. C'est le cas de l'évolution; l'intervention d'un dessein (intelligent ou non) n'est pas nécessaire pour expliquer la vie. La vie elle-même est source de vie, son action est de perpétuer la vie à travers des organismes adaptés aux conditions de leur environnement. Pour que cela dure, il est de la responsabilité des scientifiques de partager leur savoir et de ne pas le dévoyer.

\section{Références}

Bernhard, F., Coplin, D.L., et al., 1993. A gene cluster for amylovoran synthesis in Erwinia amylovora: characterization and relationship to cps genes in Erwinia stewartii, Mol. Gen. Genet., 239, 1-2, 158-168.

Bugert, P., Geider, K., 1995. Molecular analysis of the ams operon required for exopolysaccharide synthesis of Erwinia amylovora, Mol. Microbiol., 15, 5, 917-933.

Coplin, D. L., Frederick, R.D., et al., 1992. Characterization of a gene cluster that specifies pathogenicity in Erwinia stewartii, Molecular Plant-Microbe Interactions, 5, 1, 81-88.

Darwin, C., 1859. On the Origin of Species, London, John Murray. 
Delsol, M., 1997. Évolution et religions, Dossier Pour la science, 14, 12-13.

Dolph, P., Majerczak, D.R., et al., 1988. Characterization of a gene cluster for exopolysaccharide biosynthesis and virulence in Erwinia stewartii, Journal of Bacteriology, 170, 2, 865-871.

Filée, J., Forterre, P., et al., 2002. Evolution of DNA polymerase families: evidence for multiple gene exchange between cellular and viral proteins, Journal of Molecular Evolution, 54, 763-773.

Filée, J., Forterre, P., et al., 2003. The role played by viruses in the evolution of their hosts: a view based on informational protein phylogenies, Research in Microbiology,154, 237-243.

Gayon, J., 1997. Hasard et évolution, Dossier Pour la science, 14, 10-11.

Kimura, M., 1997. La théorie neutraliste de l'évolution moléculaire, Dossier Pour la science, 14, 116-123.

Klarsfeld, A., Revah, F., 2000. Biologie de la mort, Paris, Odile Jacob.

Kupiec, J.-J., Sonigo, P., 2000. Ni Dieu ni gène : pour une autre théorie de l'hérédité, Paris, Le Seuil.

Laurent, J., 1983. Are the antigenic characteristics of vesicular stomatitis virus modified during evolution in Drosophila? Ann. Virol. (Inst. Pasteur), 134 E, 465-485.

Laurent, J., 1984. Analyse du pouvoir infectant du virus de la stomatite vésiculeuse au cours de son évolution chez la drosophile, Ann. Virol. (Inst. Pasteur), 135 E, 81-87.

Laurent, J., 1997. Mutation et nouveauté, Dossier Pour la science, $14,94-97$.
Laurent, J., 2003. Le hasard novateur, Sciences et avenir, Horssérie, 134, 40-45.

Laurent, J., Lafay, F., 1983. Oligosaccharide moieties of Drosophila-adapted vesicular stomatitis virus grown in different cells, Ann. Virol. (Inst. Pasteur), 134 E, 301-308.

Luria, S.E., Delbrück, M., 1943. Mutations of bacteria from virus sensitivity to virus resistance, Genetics, 28, 491-511.

Menggad, M., Laurent, J., 1998. Mutations in ams genes of Erwinia amylovora affect the interactions with host plants, European Journal of Plant Pathology, 104, 313-322.

Philippe, H., Laurent, J., 1998. How good are deep phylogenetic trees?, Current Opinion in Genetics and Development, 8, 616623.

Philippe, H., Lopez, P., et al., 2000. Early-branching or fastevolving eukaryotes? An answer based on slowly evolving positions, Proc. R. Soc. Lond., B 267, 1213-1221.

Radman, M., et al., 2000. Evolution-driving genes, Research in Microbiology, 151, 2, 91-95.

Salomon, J.-J., 2006. Les Scientifiques entre pouvoir et savoir, Paris, Albin Michel.

Smocovitis, V.B., 1992. Unifying biology: the evolutionary synthesis and evolutionary biology, J. Hist. Biol., 25, 1, 1-65.

Sniegowski, P.D., Gerrish, P.J., et al., 2000. The evolution of mutation rates: separating causes from consequences, BioEssays, 22, 12, 1057-1066. 